\title{
Janus electrochemistry: asymmetric functionalization in one step.
}

\author{
David Ibañez, ${ }^{\dagger}$ Elisa Valles, ${ }^{\ddagger}$ Elvira Gomez, ${ }^{\dagger}$ Alvaro Colina, ${ }^{* \dagger}$ and Aranzazu Heras. ${ }^{* \dagger}$ \\ ${ }^{\dagger}$ Department of Chemistry, Universidad de Burgos, Pza. Misael Bañuelos s/n, 09001 Burgos, Spain. \\ * Grup d’Electrodeposició de Capes Primes i Nanoestructures (GE-CPN). Dep. Ciència de Materials i Química Física and \\ Institut de Nanociència i Nanotecnologia (IN2UB). Universitat de Barcelona, 08028 Barcelona, Spain.
}

\begin{abstract}
Janus structures represent an overwhelming member of materials with adaptable chemical and physical properties. The development of new synthesis routes has allowed the fabrication of Janus architectures with specific characteristics depending on the final applications. In the case of the membranes, the improvement of wet routes has been limited to the capillary effect, in which the solution can gradually penetrate through the membrane avoiding a double modification, different at each face of the membrane. In this work, we propose a new electrochemical methodology to circumvent the capillary limitation and to obtain a double electrochemical functionalization in only one step in a controlled way. This innovative methodology has been validated using a tridirectional spectroelectrochemistry setup. Moreover, the information provided by this optical arrangement should be especially useful on the study of the different processes (ion transfer, assisted ion transfer and electron transfer) that can take place at liquid/liquid interfaces. Janus electrochemistry allows us to modify the two faces of a free-standing single-walled carbon nanotube electrode in a single experiment. As proof of concept, the free-standing films have been functionalized with two different conducting polymers, polyaniline and poly(3-hexylthiophene), in one electrochemical experiment. According to the obtained results, this new electrochemical methodology will open up new gates for the design and functionalization of Janus materials.
\end{abstract}

KEYWORDS: Janus; liquid/liquid interfaces; electrochemistry; spectroelectrochemistry; ionic liquids; conducting polymers

\section{INTRODUCTION}

Over the last few decades, Janus structures have received growing attention from the scientific community due to the wide range of fascinating applications in which they offer suitable possibilities. ${ }^{1-9}$ The asymmetry of Janus membranes provides a unique factor in the development and design of new architectures with dual nature in the same structure. These novel materials can be classified according to different criteria such as their architecture and dimensionality ${ }^{10}$ composition ${ }^{10}$ or structure. ${ }^{11}$ Furthermore, the synthesis route is a key factor in the fabrication of Janus materials with distinguishing features. Different strategies have been successfully used in the fabrication of Janus membranes that can be summarized in asymmetric fabrication and asymmetric decoration. In the asymmetric fabrication each face of the membrane is previously and independently fabricated, and the Janus composite is obtained due to the combination of the two faces. Examples of this fabrication route are sequential filtration, ${ }^{12}$ sequential electrospinning ${ }^{13}$ and migration or phase separation of immiscible components in the membrane. ${ }^{14}$ On the other hand, asymmetric decoration or singleface modification consists of two steps, the first one associated with the formation of the membrane, and the second step in which Janus structure is achieved by the functionalization of the membrane. Preparation of Janus membranes by crosslinking, ${ }^{15}$ photo-degradation, ${ }^{16}$ vapor processing, ${ }^{17}$ coating, ${ }^{18}$ electrochemical deposition, ${ }^{19}$ floated deposition ${ }^{20}$ and sequential surface modification ${ }^{21}$ are examples of asymmetric decoration route. Traditionally, several drawbacks have been observed in a wet process, ${ }^{11}$ as the capillary limitation in which the solution gradually penetrates through the membrane pores leading to the same modification on both sides, i.e., different functionalization of the two faces of the membrane in a unique step becomes impossible. In addition, if the membrane surface has hydrophobic properties, the solution will be dehydrated and it leads to a nonuniform modification. In this work, we propose a new electrochemical methodology that will be the base for a new route to fabricate Janus structures by simultaneous functionalization of two-faces in a single step. The design and the fabrication of Janus architectures with specific properties using this new methodology can be extremely useful to researches ascribed to different fields such as organic chemistry, inorganic chemistry, materials science, biology, electrocatalysis, photocatalysis or bioremediation. This new methodology provides several advantages as shorter times of fabrication, but the most important improvement is that using two immiscible solutions (aqueous and hydrophobic ionic liquid) the limitation of the capillary effect in a wet process can be circumvented, leading to uniform modifications of both faces. In order to validate the new strategy to carry out electrochemical experiments, and therefore, the fabrication of Janus structures, different systems have been studied such as reversible redox couples and conducting polymers electrodeposition.

\section{EXPERIMENTAL SECTION}

Reagents and materials. Sodium hexachloroiridate (III) hydrate $\left(\mathrm{Na}_{3} \mathrm{IrCl}_{6} \cdot \mathrm{xH}_{2} \mathrm{O}, 30-36 \%\right.$, Acros Organics), lithium chloride ( $\mathrm{LiCl}, \mathrm{Merck}), 1,1$ '-dimethylferrocene (DMFc, Sigma-Aldrich), 1-butyl-1-methylpyrrolidinium bis(trifluoromethanesulfonyl)imide (BmpyrNTf 2 , Solvionic), 3-hexylthiophene (3HT, $\geq 99 \%$, Sigma Aldrich), aniline (Merck), nitric acid $\left(\mathrm{HNO}_{3}\right.$, $65 \%$, VWR) were used to prepare the solutions. All chemicals 
were of analytical grade. Aqueous solutions were freshly prepared, or stored at $4{ }^{\circ} \mathrm{C}$, using ultrapure water $(18.2 \mathrm{M} \Omega \mathrm{cm}$ resistivity at $25^{\circ} \mathrm{C}$, Milli-Q Direct 8 , Millipore).

Single-walled carbon nanotubes (SWCNTs, Sigma-Aldrich), 1,2-dichloroethane (DCE, 99.8\% for HPLC, Acros Organics), acetone (VWR), nitrocellulose membrane (filter pore size 0.45 $\mu \mathrm{m}$, Millipore), poly(ethylene terephthalate) (PET, $175 \mathrm{~mm}$ thick, HiFi Industrial Film), conductive silver paint (Electrolube) for ohmic contacts, and a high temp masking tape (Kapton) were used to fabricate the free-standing single-walled carbon nanotube (FS-SWCNT) electrodes.

Instrumentation. All electrochemical measurements were carried out at room temperature using a potentiostat/galvanostat AUTOLAB PGSTAT 20 electrochemical system. UV-Vis absorption spectroelectrochemistry measurements in normal transmission and in parallel configurations were performed using two QE65000 spectrometers (Ocean Optics). NIR absorption spectroelectrochemistry measurements in normal transmission configuration were carried out using a NIRQuest512 spectrometer (Ocean Optics). UV-Vis and NIR spectrometers were properly synchronized with the potentiostat. The light beam, supplied by a light source (Halogen HL-2000, Avantes), was conducted to the spectroelectrochemical cell by a $230 \mu \mathrm{m}$ optical fiber (Ocean Optics) or by a $100 \mu \mathrm{m}$ bare optical fiber (Ocean Optics), and collected from the spectroelectrochemical cell and led it to the spectrometer by a $200 \mu \mathrm{m}$ optical fiber probe (Avantes) or by a $100 \mu \mathrm{m}$ bare optical fiber (Ocean Optics). Morphology of the modified FS-SWCNT membranes was observed using a scanning electron microscope Field Emission JSM-7100F Analytical Microscopy.

Fabrication of the free-standing single-walled carbon nanotube (FS-SWCNT) electrodes. The approach employed in the fabrication process of FS-SWCNT electrodes is based on previous works ${ }^{19,22,23}$ and consists of seven consecutive steps. (1) The preparation of a homogeneous dispersion of SWCNTs in DCE $(5 \mathrm{mg} / \mathrm{L})$. The homogenization of SWCNT suspension is a key step of this process as the manner that inhomogeneous films will be obtained if the SWCNTs are not completely dispersed. Homogeneous dispersions are achieved using a CY-500 tipsonicator (Optic ivymen System), applying a power of $250 \mathrm{~W}$ for $10 \mathrm{~min}$ and reducing the power to $100 \mathrm{~W}$ for another $5 \mathrm{~min}$. (2) Once a homogenous dispersion is obtained, a volume of 3 $\mathrm{mL}$ of the SWCNT dispersion is filtered under vacuum using a nitrocellulose filter. (3) When PET sheets are carefully cleaned with deionized water and dried, the nitrocellulose filter with the SWCNT film is transferred to a PET sheet with a hole of $2 \mathrm{~mm}$ diameter applying a gentle pressure around the edges of the filter to improve adhesion of the SWCNT film. (4) Next, the nitrocellulose filter is completely and carefully dissolved by slow addition of acetone and, to ensure the complete removal of the filter, the SWCNT film is thoroughly rinsed with acetone for 15 min. (5) Then, the SWCNT film fixed to the PET sheet is dried at room temperature. (6) Afterwards, an ohmic contact is made using a conductive silver paint that is dried in an oven at $75^{\circ} \mathrm{C}$ for $30 \mathrm{~min}$. (7) Finally, the ohmic contact is isolated using a high temperature masking tape.

FS-SWCNT film has a geometrical area of $0.550 \pm 0.006 \mathrm{~cm}^{2}$ in side A, delimited by the high temp masking tape used to isolate the ohmic contact, and $0.090 \pm 0.006 \mathrm{~cm}^{2}$ in side $\mathrm{B}$, delimited by the diameter of the hole $(2 \mathrm{~mm})$. According to our previous work, ${ }^{19}$ physical stability and transparency required to perform the spectroelectrochemical measurements in normal transmission configuration are inversely proportional factors. In this regard, FS-SWCNT films prepared with 2, 3 and $4 \mathrm{~mL}$ of the SWCNT dispersion filtered were analyzed, obtaining that the simultaneous long-term stability and good transparency of this membrane was obtained with FS-SWCNT films prepared with $3 \mathrm{~mL}$ of the SWCNT dispersion. Thickness of the $3 \mathrm{~mL}$ film was determined by AFM, obtaining a value of $570 \pm 30 \mathrm{~nm} .{ }^{19}$

Tridirectional spectroelectrochemistry setup. The setup employed in this work involves a free-standing single-walled carbon nanotube working electrode (FS-SWCNT-WE), a homemade $\mathrm{Ag} / \mathrm{AgCl} / \mathrm{KCl}(3 \mathrm{M})$ reference electrode ( $\mathrm{RE})$ and a counter electrode $(\mathrm{CE})$ forked in two Pt wires. The functionalization of the FS-SWCNT film was followed by spectroelectrochemistry, a hybrid technique that provides suitable, simultaneous and in-situ electrochemical and spectroscopic information. Furthermore, it has been developed a new tridirectional spectroelectrochemistry setup (Figure 1) that is based on a bidimensional setup previously proposed ${ }^{24}$. This tridirectional setup is undoubtedly very useful to study electrochemical processes in two different phases, as for example at the liquid/liquid interface. This new setup allows us to record simultaneously one optical signal in normal transmission configuration and other two in parallel configuration, one from each face of the FS-SWCNTWE. In normal transmission configuration, the $230 \mu \mathrm{m}$ optical fiber from the light source $\left(\mathrm{F}_{\mathrm{N} 1}\right)$ is connected with a collimating lens for normal transmission configuration measurements. A $200 \mu \mathrm{m}$ optical fiber probe $\left(\mathrm{F}_{\mathrm{N} 2}\right)$ is placed perpendicular to the WE to collect the light beam that passes through FS-SWCNT membrane from the light source. In parallel arrangement, on each face of the FS-SWCNT-WE, one $100 \mu \mathrm{m}$ bare optical fiber $\left(\mathrm{F}_{\mathrm{A} 1}\right.$ and $\left.\mathrm{F}_{\mathrm{B} 1}\right)$ that leads the light beam from the light source is accurately aligned with a second $100 \mu \mathrm{m}$ bare optical fiber $\left(\mathrm{F}_{\mathrm{A} 2}\right.$ and $\mathrm{F}_{\mathrm{B} 2}$ ) that collects the transmitted light beam to the spectrometer. In the two faces, the light beam in parallel configuration passes parallel and close to the electrode surface, sampling only the first $100 \mu \mathrm{m}$ of solution adjacent to the FS-SWCNT-WE. The $100 \mu \mathrm{m}$ bare optical fibers are properly fixed to both sides of the FS-SWCNT-WE at a known distance. An advantage of this novel tridirectional setup is that it allows us to adapt the optical fibers diameter and the distance between the fibers to the studied system.

Safety considerations. All handling and processing were performed carefully, particularly when DCE was used. SWCNT dispersions were stored in tempered glass material, nitrogen flushed and film coated. 


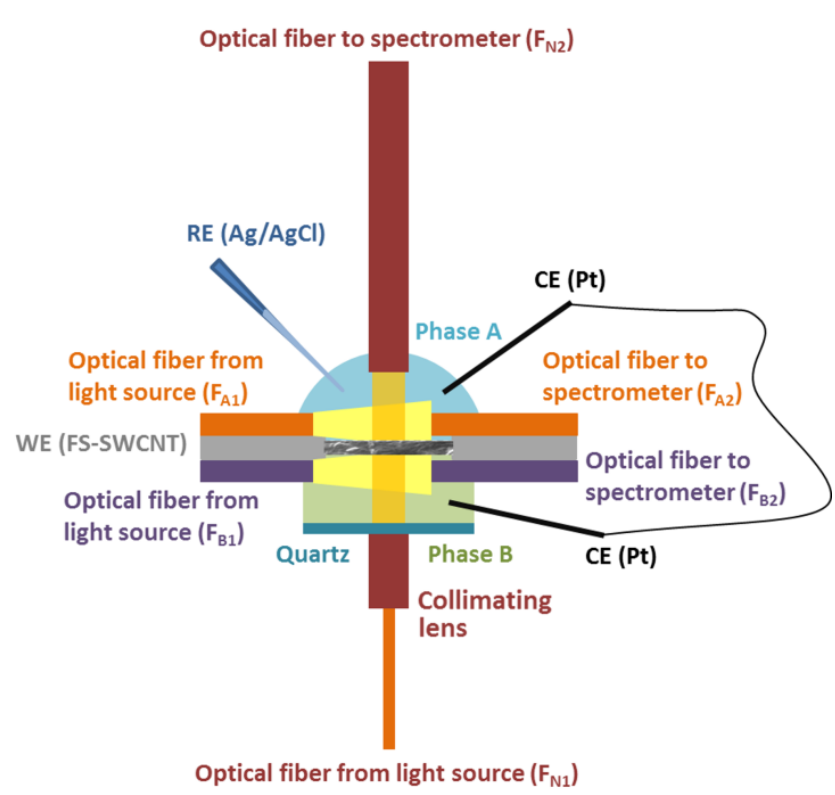

Figure 1. Detailed schematic view of the tridirectional setup, in which one spectroscopic signal in normal transmission configuration and two parallel signals, one on each face of the FS-SWCNT membrane, are recorded simultaneously during the electrochemical experiment.

\section{RESULTS AND DISCUSSION}

Validation of the electrochemical methodology using a tridirectional spectroelectrochemistry setup. As a proof of concept, two reversible redox couples widely used in electrochemistry were selected to validate the performance of the Janus electrochemistry experiment using the tridirectional spectroelectrochemistry setup. The composition used was $5 \times 10^{-3} \mathrm{M}$ $\mathrm{IrCl}_{6}{ }^{3-}$ in $0.1 \mathrm{M} \mathrm{LiCl}$ (aq) in the phase $\mathrm{A}$ and $5 \times 10^{-3} \mathrm{M} \mathrm{DMFc}$ in $\mathrm{BmpyrNTf}_{2}$ in the phase $\mathrm{B}$. Under these experimental conditions, formal potentials of $\mathrm{DMFc}^{+} / \mathrm{DMFc}$ in $\mathrm{BmpyrNTf}_{2}$ and $\mathrm{IrCl}_{6}{ }^{2-} / \mathrm{IrCl}_{6}{ }^{3-}$ in $\mathrm{LiCl}$ obtained were $+0.147 \mathrm{~V}$ and $+0.844 \mathrm{~V}$ vs $\mathrm{Ag} / \mathrm{AgCl}$, respectively. Electron transfer process was studied by chronopotentiometry, applying an anodic current, of $3 \times 10^{-5} \mathrm{~A}$ during 30 seconds (Figure $2 \mathrm{a}$ ). In all cases, the spectrum of each phase before applying the electrical current was taken as reference. Initially, DMFc and $\mathrm{IrCl}_{6}{ }^{3-}$ are present in their reduced forms, so only the oxidation to $\mathrm{DMFc}^{+}$and $\mathrm{IrCl}_{6}{ }^{2-}$ can take place, independently in each phase. Figure $2 \mathrm{~b}$ displays the UVVis spectra in parallel configuration in the DMFc phase (blue lines) and in the iridium aqueous phase (red lines). As can be observed, UV-Vis spectra of DMFc in phase B display two characteristic bands at 290 and $650 \mathrm{~nm}$, while UV-Vis spectra of $\mathrm{IrCl}_{6}{ }^{3-}$ in phase A show three distinctive bands at 430, 490 and $580 \mathrm{~nm}$, ascribed to the oxidized forms of DMFc and $\mathrm{IrCl}_{6}{ }^{3-}$ respectively. ${ }^{23}$ The integration times for UV-Vis spectra were $200 \mathrm{~ms}$ in the phase A and $100 \mathrm{~ms}$ in the phase B. These values indicate the high time-resolved data acquisition offered by this experimental setup.

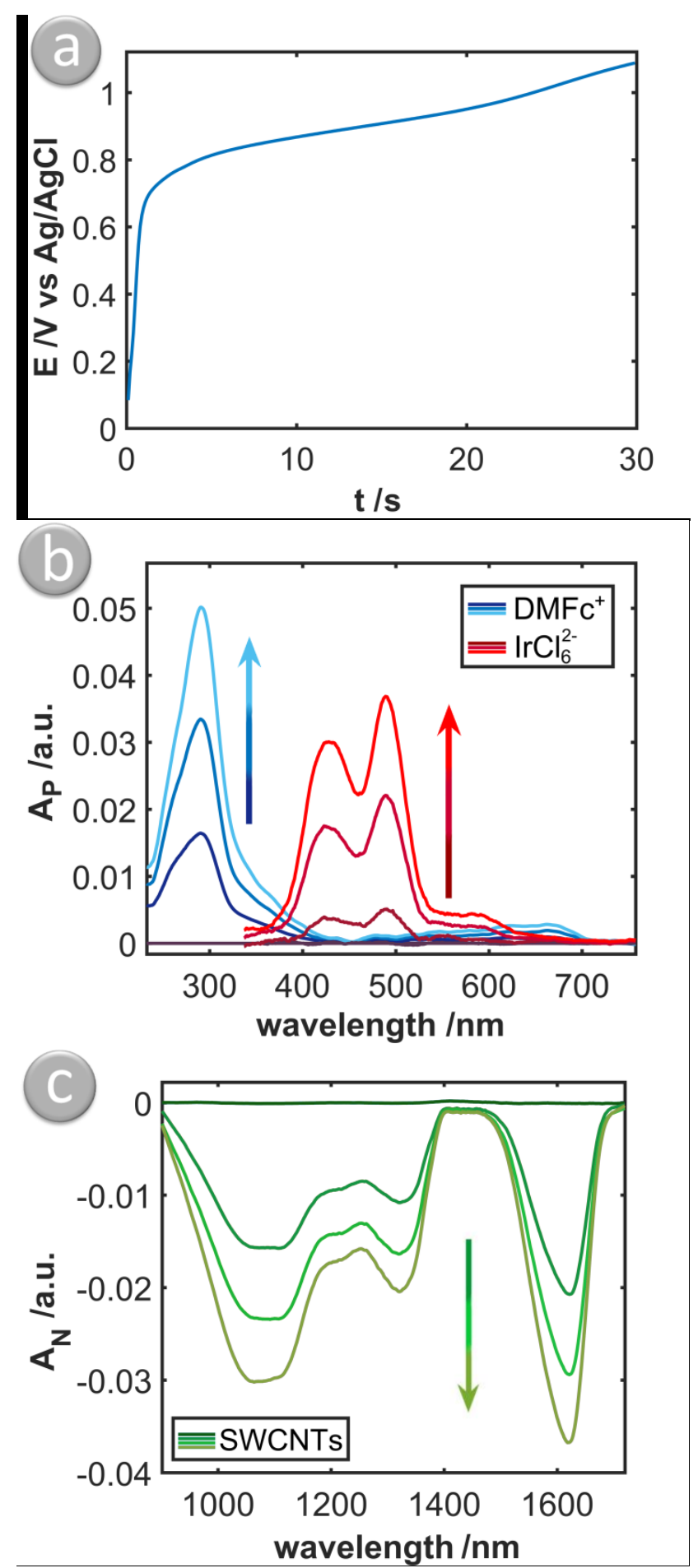

Figure 2. (a) Chronopotentiogram at $3 \times 10^{-5} \mathrm{~A}$, (b) UV-Vis and (c) NIR spectra recorded at $0,8,16$ and $24 \mathrm{~s}$ in parallel (Ap) and normal transmission $\left(\mathrm{A}_{\mathrm{N}}\right)$ configuration, respectively. Integration time for UV-Vis spectra: $200 \mathrm{~ms}$ in the phase A and $100 \mathrm{~ms}$ in the phase B. Integration time for NIR spectra: $300 \mathrm{~ms}$. The chemical composition of the solutions used was $5 \cdot 10^{-3} \mathrm{M} \mathrm{IrCl}_{6}{ }^{3-}$ and $0.1 \mathrm{M} \mathrm{LiCl}(\mathrm{aq})$ in the phase A and $5 \cdot 10^{-3} \mathrm{M}$ DMFc in BmpyrNTf $_{2}$ in the phase B.

Time-resolved evolution of absorbance at $290 \mathrm{~nm}$ for DMFc and at 430 and $490 \mathrm{~nm}$ for iridium is shown in Figure 3a and Figure 3b, respectively. Chronoabsorptogram at $290 \mathrm{~nm}$ (related to DMFc, Figure 3a) shows the progressive absorbance 
increase during the chronopotentiometry, i.e., when the oxidation of $\mathrm{DMFc}$ to $\mathrm{DMFc}^{+}$takes place. Figure $3 \mathrm{~b}$ illustrates a similar behavior in the chronoabsorptograms at 430 and $490 \mathrm{~nm}$ associated with the iridium couple, showing that the oxidation of $\mathrm{IrCl}_{6}{ }^{3-}$ to $\mathrm{IrCl}_{6}{ }^{2-}$ also produces the increase of absorbance. UV-Vis absorption signals in parallel arrangement confirm that the two oxidation processes occur concomitantly and independently in the two phases (Figures 2b, 2c, 3a and 3b).
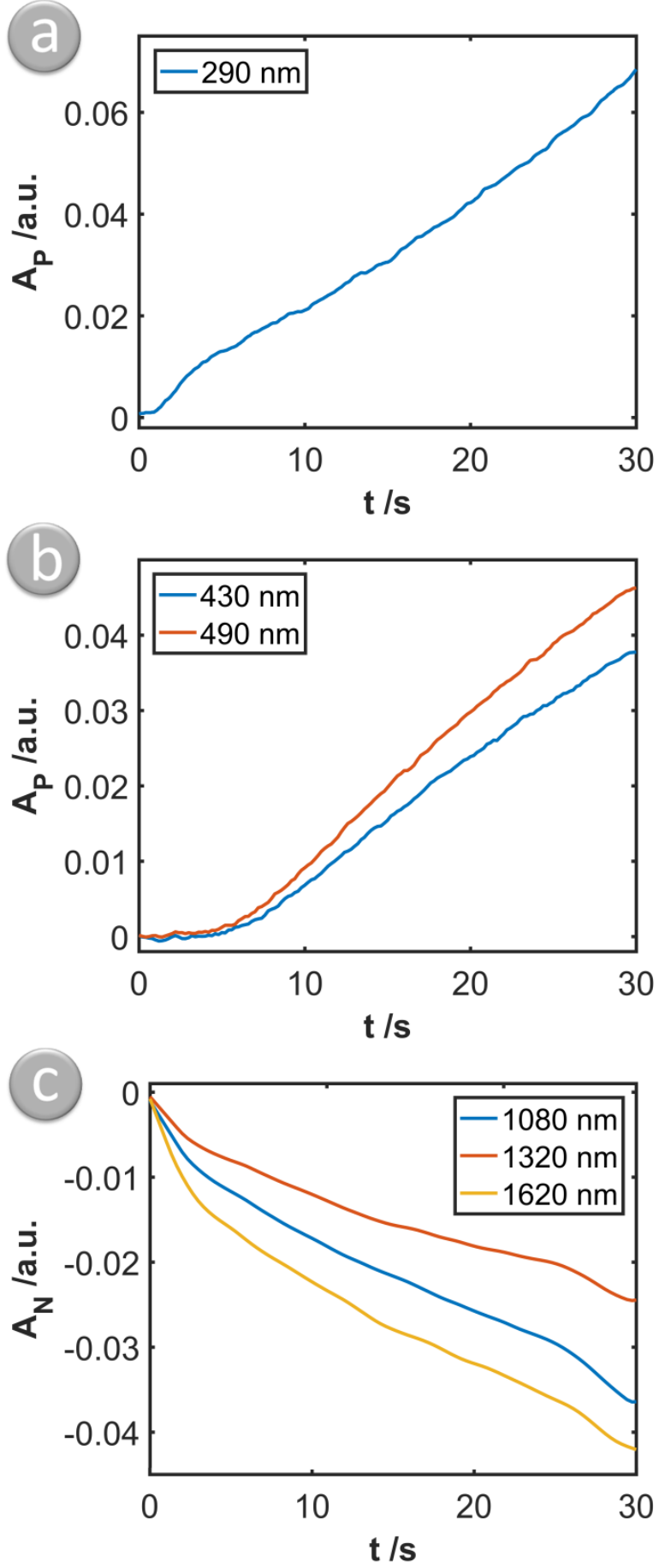

Figure 3. Chronoabsorptograms at characteristic UV-Vis wavelengths of (a) DMFc in BmpyrNTf 2 and (b) $\mathrm{IrCl}_{6}^{3-}$ in $0.1 \mathrm{M} \mathrm{LiCl}$ (aq) recorded in parallel transmission configuration. (c) Chronoabsorptograms at 1080, 1320 and $1620 \mathrm{~nm}$, characteristic NIR bands of SWCNTs recorded in normal transmission configuration. The chemical composition of the solutions used was the same detailed in Figure 2.
The third optical signal is recorded simultaneously in normal configuration (integration time $300 \mathrm{~ms}$ ). In this case, Figure 2c displays NIR spectra in which the typical bands of the SWCNTs are observed. Chronoabsorptograms of the bands peaking at 1080, 1320 and $1620 \mathrm{~nm}$, Figure 3c, show that the absorbance in the NIR region decreases with time due to changes in the redox state of the SWCNTs of the membrane during the electrochemical experiment.

In order to establish if the behavior of these NIR bands depends on the oxidation of DMFc and $\mathrm{IrCl}_{6}{ }^{3-}$ in solution or only on processes occurring in the FS-SWCNT membrane, a blank experiment was performed without soluble redox species in neither of the two phases. A chronopotentiometry at $3 \times 10^{-5}$ A during 30 $\mathrm{s}$ with $0.1 \mathrm{M} \mathrm{LiCl}(\mathrm{aq})$ in the phase $\mathrm{A}$ and $\mathrm{BmpyrNTf}_{2}$ in the phase B was carried out. Electrochemical response (Figure S1a) shows that the potential reached at the end of the experiment is more positive than the potential value observed when DMFc and $\mathrm{IrCl}_{6}{ }^{3-}$ are present in the different phases (Figure 2a). Analyzing NIR spectra and according to the literature, ${ }^{25}$ we can conclude that the NIR bands observed in Figure $2 \mathrm{c}$ and in the blank experiment (Figure S1b) are exactly the same, and hence they are only related to oxidation of SWCNTs. Finally, the same experiment displayed in Figure 2 (composition of the cell: $5 \times 10^{-}$ ${ }^{3} \mathrm{M} \mathrm{IrCl}_{6}{ }^{3-}$ in $0.1 \mathrm{M} \mathrm{LiCl}(\mathrm{aq})$ in the phase A and $5 \times 10^{-3} \mathrm{M}$

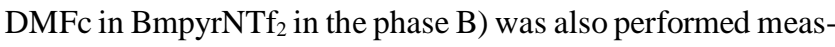
uring in this case the UV-Vis spectra (Figure S2) in normal arrangement, emerging only the bands related to $\mathrm{SWCNTs}^{26}$ because of the lower sensitivity in normal configuration compared with that in parallel arrangement.

From the results shown in this section, we can conclude that tridirectional spectroelectrochemistry confirms the independent electrochemical reactions at the two faces of the FS-SWCNT film. Therefore, Janus electrochemistry should be useful for the independent modification of the two faces of conductive membranes with different materials in a unique step.

Asymmetric Janus functionalization of FS-SWCNT membranes. Once the new electrochemical approach was validated with two reversible redox couples, the simultaneous functionalization of both sides of the FS-SWCNT membrane was performed by the electrodeposition of two different conducting polymers, in particular polyaniline (PANI) and poly(3-hexylthiophene) (P3HT). The composition used was $0.1 \mathrm{M}$ aniline and $0.5 \mathrm{M} \mathrm{HNO}_{3}(\mathrm{aq})$ in the phase $\mathrm{A}$ and $0.1 \mathrm{M}$ 3-hexylthiophene (3HT) in BmpyrNTf 2 in the phase B. Taking into account that in these experiments the modification takes place on the two surfaces of the FS-SWCNT membrane, the spectroelectrochemistry measurements were only performed in normal configuration.

Electrochemical functionalization was performed by applying 5 $\times 10^{-4}$ A during $100 \mathrm{~s}$ (Figure 4a) to generate the corresponding polymer at each face. Figure $4 \mathrm{~b}$ shows the UV-Vis spectra in normal configuration. Three absorption bands are observed, two associated with PANI and a third one with P3HT. In particular, the band at $425 \mathrm{~nm}$ is mainly related to the PANI oligomers, and the band at $710 \mathrm{~nm}$ is associated to the electrodeposited PANI film on the face A of the membrane, ${ }^{27,28}$ while the absorption band at $550 \mathrm{~nm}$ is related to the $\mathrm{P} 3 \mathrm{HT}$ electrodeposited in the face B of the FS-SWCNT electrode. ${ }^{29-31}$ 

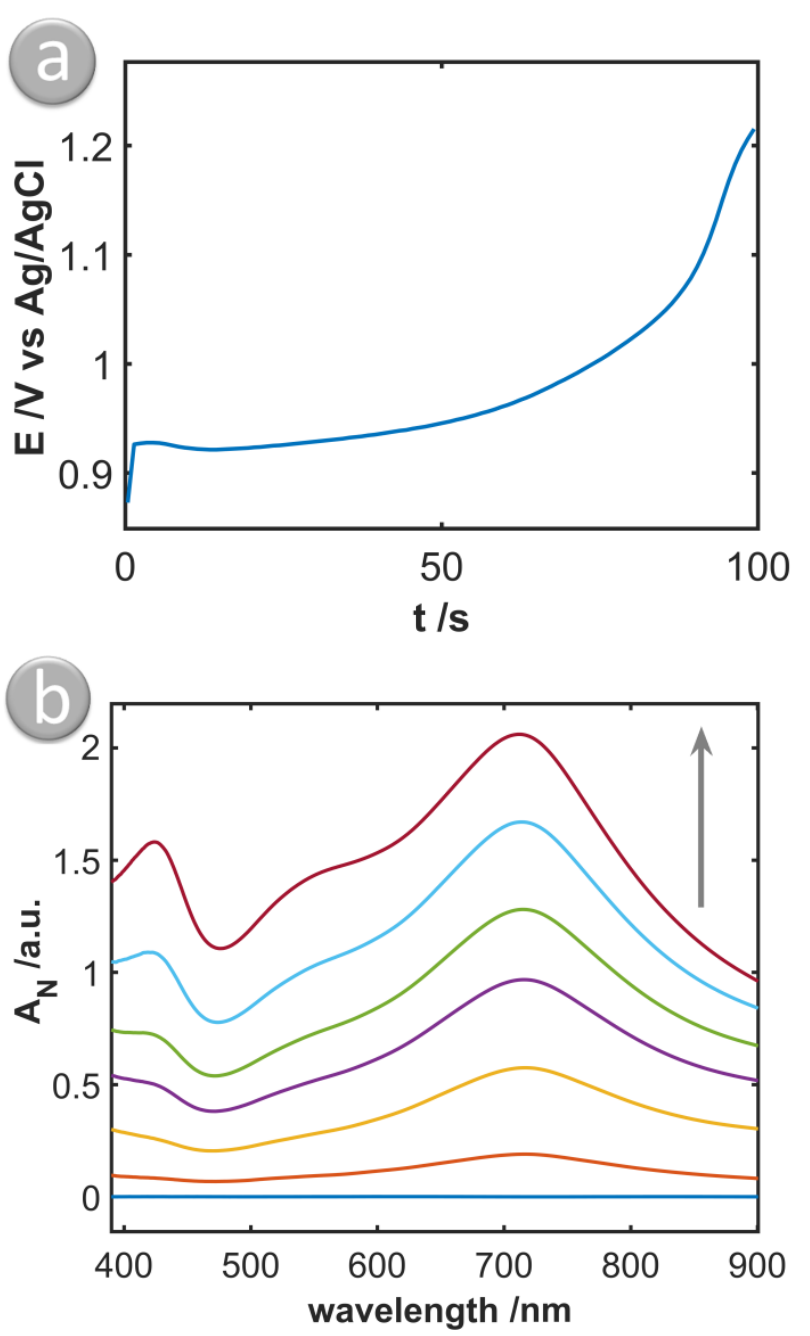

Figure 4. (a) Chronopotentiogram at $5 \times 10^{-4} \mathrm{~A}$, and (b) UV-Vis absorption spectra recorded in normal transmission configuration during the electrodeposition of PANI and P3HT on each face of the FS-SWCNT membrane. Integration time for UV-Vis spectra: 20 $\mathrm{ms}$. The chemical composition of the solutions used was $0.1 \mathrm{M}$ aniline and $0.5 \mathrm{M} \mathrm{HNO}_{3}(\mathrm{aq})$ in the phase $\mathrm{A}$ and $0.1 \mathrm{M} 3 \mathrm{HT}$ in BmpyrNTf $_{2}$ in the phase $\mathrm{B}$.

Figure S3a shows the UV-Vis spectra of PANI and P3HT electrodeposited independently on two different SWCNT electrodes: the absorption spectrum of PANI electrogenerated on a SWCNT electrode shows a band at $710 \mathrm{~nm}$, while the characteristic UV-Vis spectrum of P3HT synthesized on a different SWCNT electrode displays a band at $550 \mathrm{~nm}$. Comparison between spectra in Figure S3a and those in Figure 4b demonstrates that UV-Vis spectra of PANI and P3HT electrosynthesized on different electrodes agree with the absorption spectra of the two polymers generated in a unique experiment.

Evolution of absorbance with time at the two characteristic wavelengths of the polymers, 710 and $550 \mathrm{~nm}$, is illustrated in Figure 5. Although in both cases, the absorbance increases progressively during the galvanostatic experiment, the highest increase of absorbance is observed during the last $30 \mathrm{~s}$ when potential reaches more positive values (Figure 4a). Thus, these results confirm that PANI and P3HT have been synthesized simultaneously in one step.
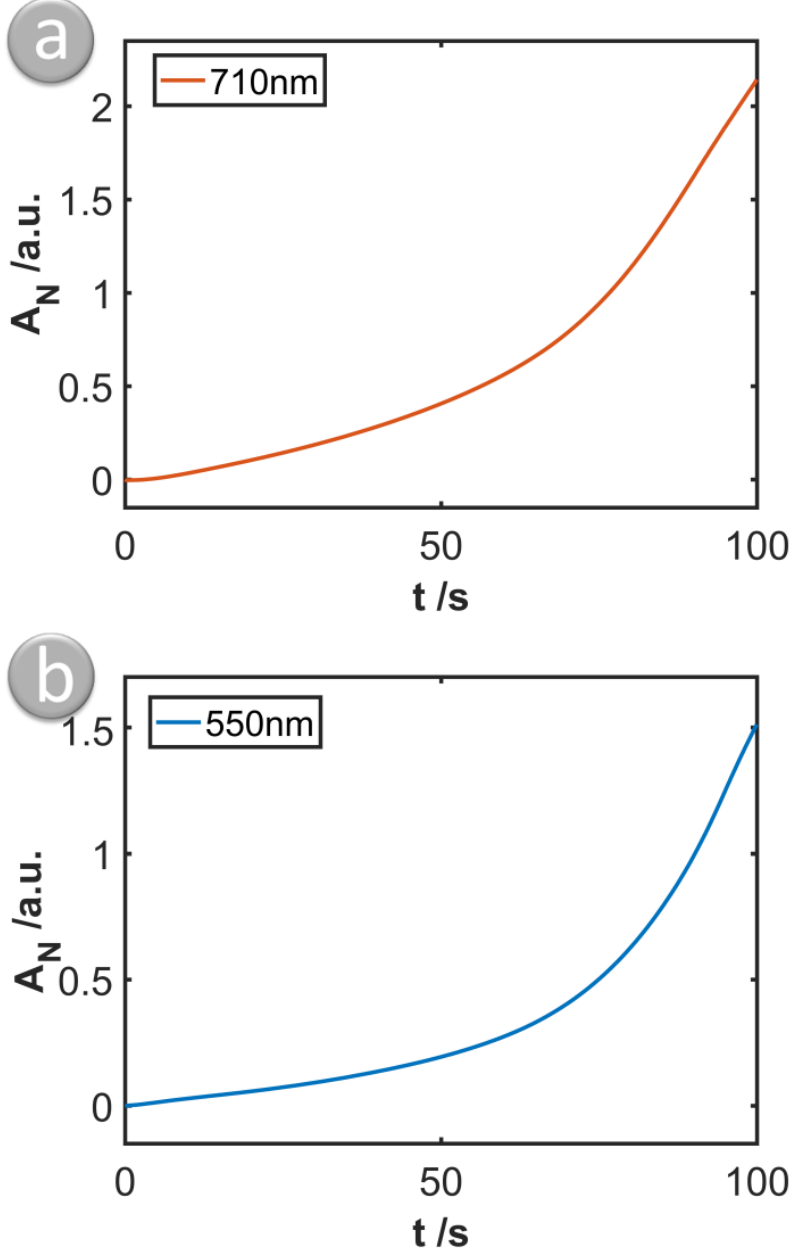

Figure 5. Chronoabsorptograms at (a) 710 and (b) $550 \mathrm{~nm}$, maximum wavelengths of the absorption bands related to PANI and $\mathrm{P} 3 \mathrm{HT}$, respectively. The chemical composition of the solutions was the same detailed in Figure 4.

Different experimental conditions were tested, and for example, the dependence of the electropolymerization process on the applied current was analyzed. Figure S4a displays the chronopotentiometry at $5 \times 10^{-5} \mathrm{~A}$. As can be noticed in Figure 6a, the $\mathrm{UV}-\mathrm{V}$ is spectra do not show the characteristic absorption bands of PANI and P3HT, and only the bands related to the SWCNTs of the FS membrane are observed. Comparison between the $\mathrm{UV}-\mathrm{V}$ is spectra recorded applying a current intensity of $5 \times 10^{-}$ ${ }^{5} \mathrm{~A}$ (Figure 6a) and $5 \times 10^{-4} \mathrm{~A}$ (Figure 4b) during $100 \mathrm{~s}$, allows us to conclude that at the lowest current intensity the electrosynthesis of the conducting polymers does not occur. Applying a current intensity of $1 \times 10^{-4}$ A (Figure S4b) only the electrosynthesis of PANI occurs. In this case, the UV-Vis spectra (Figure 6b) show the characteristic absorption band of the PANI at $710 \mathrm{~nm}$ but the P3HT band at $550 \mathrm{~nm}$ is not observed. Hence, the current intensity applied produces the electropolymerization of aniline but is not enough to cause the electrogeneration of P3HT. According to the literature, electrosynthesis of PANI in $\mathrm{HNO}_{3}$ takes place around $+0.90 \mathrm{~V},{ }^{27}$ while the generation of $\mathrm{P} 3 \mathrm{HT}$ in BmpyrNTf $\mathrm{B}_{2}$ occurs at more positive potential, +1.35 $\mathrm{V}^{32}$ In this way, UV-Vis absorption spectra observed in Figure $4 \mathrm{~b}$, applying a current intensity of $5 \times 10^{-4} \mathrm{~A}$, agree with the oxidative potentials of PANI and P3HT, because the potential 
did not reach the value required to form P3HT and only PANI was generated.
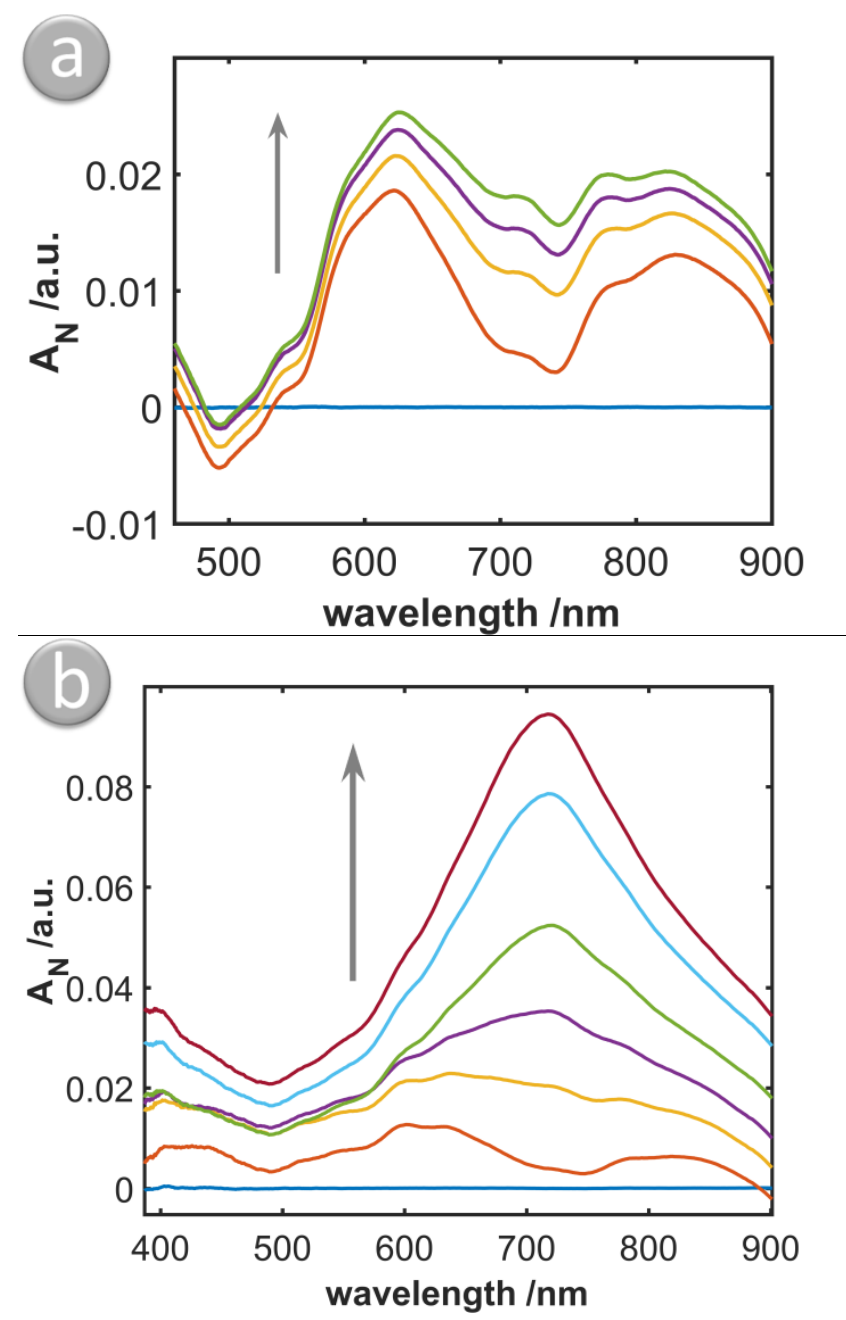

Figure 6. UV-Vis absorption spectra recorded in normal transmission configuration during the chronopotentiometry at (a) $5 \times 10^{-5}$ (integration time $20 \mathrm{~ms}$ ) and (b) $1 \times 10^{-4} \mathrm{~A}$ (integration time $20 \mathrm{~ms}$ ). The chemical composition of the solutions used was $0.1 \mathrm{M}$ aniline and $0.5 \mathrm{M} \mathrm{HNO}_{3}(\mathrm{aq})$ in the phase A and $0.1 \mathrm{M} 3 \mathrm{HT}$ in $\mathrm{BmpyrNTf}_{2}$ in the phase $\mathrm{B}$.

Finally, the composition of the chemical system was modified by increasing the $3 \mathrm{HT}$ concentration to $0.2 \mathrm{M}$, keeping the aniline concentration $(0.1 \mathrm{M})$ and the current intensity $\left(5 \times 10^{-4} \mathrm{~A}\right)$ constant. In this case, the UV-Vis spectra (Figure S5b) show not only that both polymerization processes have taken place because the evolution of the characteristic absorption bands of PANI and P3HT are observed, but also that the absorbance ratio, $\mathrm{A}_{710 \mathrm{~nm}} / \mathrm{A}_{550 \mathrm{~nm}}=1.29$, changes respect to Figure $4 \mathrm{~b}$ when the concentration of $3 \mathrm{HT}$ in $\mathrm{BmpyrNTf}_{2}$ is lower, 0.1 M ( $\mathrm{A}_{710}$ $\left.\mathrm{nm} / \mathrm{A}_{550 \mathrm{~nm}}=1.44\right)$. As can be noticed in the comparison between Figure 4b and Figure S5b, absorbance of band at $550 \mathrm{~nm}$, related to P3HT, is higher when the concentration of $3 \mathrm{HT}$ is 0.2 $\mathrm{M}$. On the other hand, absorbance at $710 \mathrm{~nm}$ associated with PANI maintains similar value because the initial concentration of aniline was not modified.

Hence, the control of the applied current intensity and/or the monomers concentrations in the experiment allows the tailoring of the fabrication of Janus membranes with specific composition. Figure S5c and Figure S5d show the chronoabsorptograms at 710 and $550 \mathrm{~nm}$, respectively. Evolution of absorbance values with time at these wavelengths demonstrates as in the previous case (Figure 5) that although absorbance values increase progressively during the galvanostatic experiment, the highest increase is observed during the last $30 \mathrm{~s}$ when the potential reaches more positive values. In particular, absorbance at 550 $\mathrm{nm}$ reaches a higher value because more P3HT was electrogenerated under these experimental conditions.

The characterization of each face of the electrochemically modified FS-SWCNT membrane was performed by scanning electron microscopy (SEM) and by cyclic voltammetry. Figure 7a and Figure $7 \mathrm{~b}$ display SEM images of PANI and P3HT faces, respectively. As can be observed, the morphology of each face is completely different: PANI forms a homogeneous and continuous coating film on the SWCNTs (Figure 7a) while P3HT forms agglomerates with granular morphology (Figure 7b). Although PANI film is not easily distinguished in the SEM images because of its homogeneity, inset in Figure 7a shows clearly the PANI coating on the SWCNT film. SEM images of the unmodified FS-SWCNT membrane, the side of the film modified with PANI and the face of the film modified with P3HT are shown in Figure S6. Comparison between the unmodified membrane (Figure S6a) and the side of the film modified with PANI (Figure S6b) evidences PANI coating formed on the SWCNT, more complicated to distinguish than P3HT agglomerates (Figure S6c). Taking into account that SEM images are not sufficient to establish distinct surface modification, the independent electrochemical characterization of each side of the functionalized FS membrane was carried out cycling the electrode potential four times. Electrochemical routes are one of the most powerful techniques used to evaluate the Janus architectures obtained by this innovative one step method. PANI characterization was performed from $-0.10 \mathrm{~V}$ to $+0.90 \mathrm{~V}$ and back to $-0.10 \mathrm{~V}$ at 0.05 $\mathrm{V} \mathrm{s}^{-1}$ in $0.5 \mathrm{M} \mathrm{HNO}_{3}$ (aq). Figure 7c (blue line) displays the cyclic voltammogram, in which the three characteristic reversible pairs of peaks related to the different doped forms of PANI can be distinguished at potentials around $+0.21 \mathrm{~V},+0.53 \mathrm{~V}$ and $+0.75 \mathrm{~V} .{ }^{27}$ Figure $7 \mathrm{c}$ (orange line) shows the cyclic voltammogram for the P3HT face. In this case, the electrochemical characterization was performed between $-0.10 \mathrm{~V}$ and $+1.30 \mathrm{~V}$ at $0.05 \mathrm{~V} \mathrm{~s}^{-1}$ in BmpyrNTf $\mathrm{B}_{2}$. Electrochemical response is clearly different respect to the PANI one and only one peak associated with P3HT doping is observed at around $+1.00 \mathrm{~V} .{ }^{33}$ Hydrophobic properties of FS-SWCNT membranes allow the independent characterization of each face. Comparison between Figure 7 and Figure S3b, that displays the electrochemical characterization of PANI and P3HT electrogenerated using two different SWCNT electrodes, demonstrates not only that both polymers are formed, but also that the independent and simultaneous electropolymerization of different monomers on each face of the FS-SWCNT substrate. 

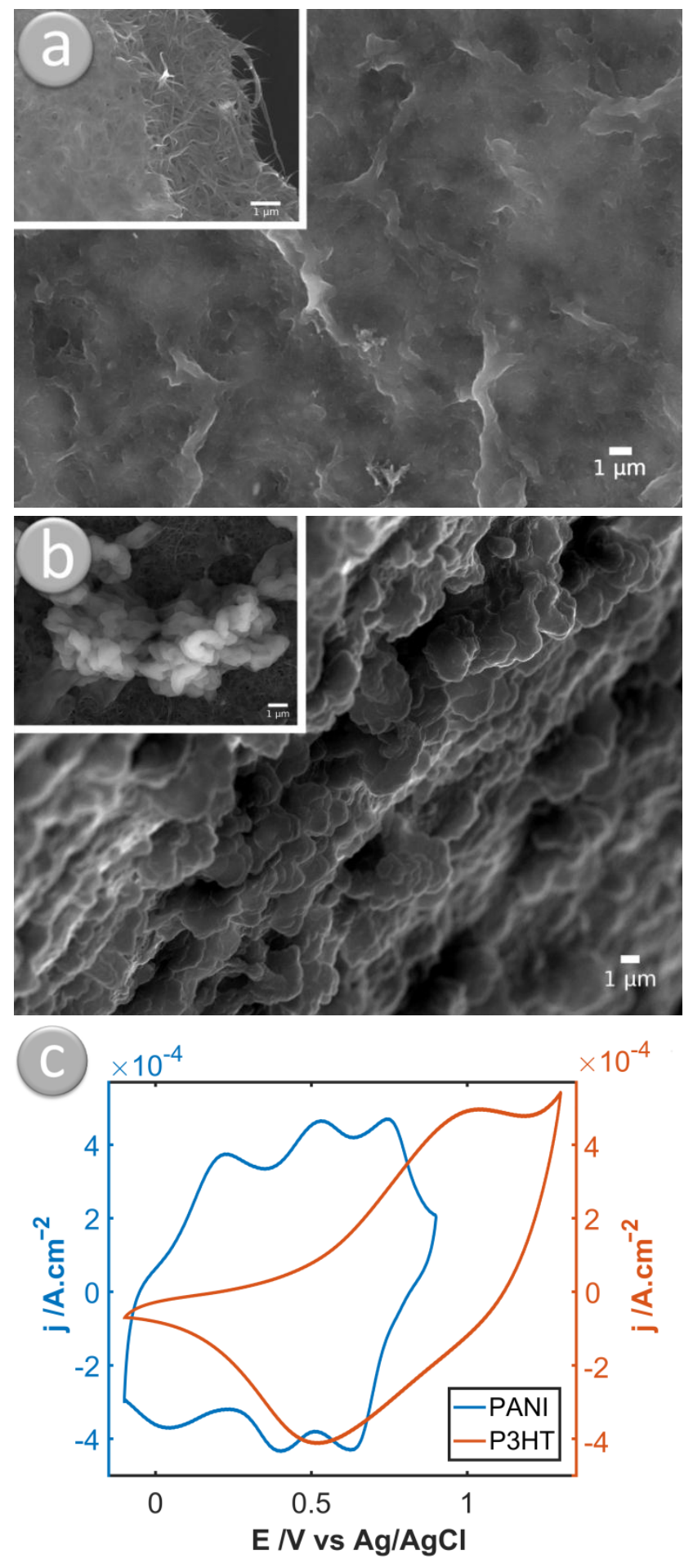

Figure 7. SEM image of (a) PANI and (b) P3HT faces, respectively. Insets show more details of each polymer electrogenerated. (c) Cyclic voltammetric characterization of PANI (blue line) and P3HT (orange line) faces.

\section{CONCLUSIONS}

In conclusion, a novel strategy to fabricate FS-SWCNT Janus membranes has been developed. This methodology allows us to circumvent the capillary limitation in wet processes. Moreover, the validation of this electrochemical method has entailed the development of a new tridirectional setup that provides one electrochemical response and three spectroscopic signals concomitantly and independently. This novel device enables the analysis of complex processes that take place simultaneously on each face of the membrane, being especially useful to study complex electrochemical systems as liquid/liquid interfaces. Quite importantly, Janus structures based on FS-SWCNT films are achieved by a new electrochemical strategy, getting the double functionalization in one unique step. In this work, the nature of each face of the FS-SWCNT film has been independently modified with PANI and P3HT. Double functionalization has been demonstrated by SEM images and cyclic voltammetry. This approach provides the basis for new electrochemical routes to design and fabricate Janus architectures with specific properties.

\section{ASSOCIATED CONTENT}

\section{Supporting Information.}

This material is available free of charge via the Internet at http://pubs.acs.org.

Chronopotentiogram and NIR spectra of the blank experiment recorded in normal transmission configuration; chronopotentiogram and UV-Vis spectra recorded in normal transmission configuration; characteristic UV-Vis absorption spectra and the electrochemical characterization of PANI and P3HT electrodeposited independently on different SWCNT electrodes; chronopotentiograms at different currents; chronopotentiogram, UV-Vis absorption spectra and chronoabsorptograms using different chemical composition of the cell; SEM images of FS-SWCNT film unmodified and electrochemically modified with PANI and P3HT.

\section{AUTHOR INFORMATION}

\section{Corresponding Author}

*E-mail: acolina@ubu.es

*E-mail: maheras@ubu.es

Tel: +34 9472588 17. Fax: +34947258831.

\section{ORCID}

Alvaro Colina: 0000-0003-0339-356X

Aranzazu Heras: 0000-0002-5068-2164

\section{Author Contributions}

The manuscript was written through contributions of all authors. All authors have given approval to the final version of the manuscript.

\section{Notes}

The authors declare no competing financial interest.

\section{ACKNOWLEDGMENT}

Financial support from Ministerio de Economía y Competitividad (CTQ2014-61914-EXP, CTQ2014-55583-R， TEC2014-51940C2-2R, CTQ2015-71955-REDT) and Junta de Castilla y León (BU033-U16) is gratefully acknowledged. D.I. thanks MINECO for his postdoctoral fellowship (CTQ2014-61914-EXP). Jose Manuel Díez is acknowledged for his help in the fabrication of the electrodes (contract funded by the European Social Fund and the Youth Employment Initiative). Jesus Garcia de la Fuente (Gomensoro, Metrohm) is acknowledged for the helpful electrochemical discussions. The authors thank the CCiT-UB for the use of their equipment. 


\section{REFERENCES}

(1) Peng, H.; Bi, S.; Ni, M.; Xie, X.; Liao, Y.; Zhou, X.; Xue, Z.; Zhu, J.; Wei, Y.; Bowman, C. N.; Mai, Y-W. Monochromatic Visible Light "Photoinitibitor": Janus-Faced Initiation and Inhibition for Storage of Colored 3D Images. J. Am. Chem. Soc. 2014, 136, 8855-8858.

(2) Hu, J.; Zhou, S.; Sun, Y.; Fang, X.; Wu, L. Fabrication, Properties and Applications of Janus Particles. Chem. Soc. Rev. 2012, 41, 4356-4378.

(3) Li, J.; Hong, C.-Y.; Wu, S.-X.; Liang, H.; Wang, L.-P.; Huang, G.; Chen, X.; Yang, H.-H.; Shangguan, D.; Tan, W. Facile Phase Transfer and Surface Biofunctionalization of Hydrophobic Nanoparticles Using Janus DNA Tetrahedron Nanostructures. $J$. Am. Chem. Soc. 2015, 137, 11210-11213.

(4) Dong, R.; Hu, Y.; Wu, Y.; Gao, W.; Ren, B.; Wang, Q.; Cai, Y. Visible-Light-Driven BiOI-Based Janus Micromotor in Pure Water. J. Am. Chem. Soc. 2017, 139, 1722-1725.

(5) Pavlick, R. A.; Sengupta, S.; McFadden, T.; Zhang, H.; Sen, A A Polymerization-Powered Motor. Angew. Chemie - Int. Ed. 2011, 50, 9374-9377.

(6) Xuan, M.; Wu, Z.; Shao, J.; Dai, L.; Si, T.; He, Q. Near Infrared Light-Powered Janus Mesoporous Silica Nanoparticle Motors. J. Am. Chem. Soc. 2016, 138, 6492-6497.

(7) Millman, J. R.; Bhatt, K. H.; Prevo, B. G.; Velev, O. D. Anisotropic Particle Synthesis in Dielectrophoretically Controlled Microdroplet Reactors. Nat. Mater. 2005, 4, 98-102.

(8) Volpe, G.; Buttinoni, I.; Vogt, D.; Kummerer, H.-J.; Bechinger, C. Microswimmers in Patterned Environments. Soft Matter 2011, 7, 8810-8815.

(9) Percec, V.; Imam, M. R.; Peterca, M.; Leowanawat, P. SelfOrganizable Vesicular Columns Assembled from Polymers Dendronized with Semifluorinated Janus Dendrimers Act As Reverse Thermal Actuators. J. Am. Chem. Soc. 2012, 134, 44084420.

(10) Pang, X.; Wan, C.; Wang, M.; Lin, Z. Strictly Biphasic Soft and Hard Janus Structures: Synthesis, Properties, and Applications. Angew. Chemie Int. Ed. 2014, 53, 5524-5538.

(11) Yang, H.; Hou, J.; Chen, V.; Xu, Z. Janus Membranes : Exploring Duality for Advanced Separation Minireviews. Angew. Chemie Int. Ed. 2016, 55, 13398-13407.

(12) Hu, L.; Gao, S.; Zhu, Y.; Zhang, F.; Jiang, L.; Jin, J. An Ultrathin Bilayer Membrane with Asymmetric Wettability for Pressure Responsive Oil/water Emulsion Separation. J. Mater. Chem. A 2015, 3, 23477-23482.

(13) Lv, N.; Ma, Q.; Dong, X.; Wang, J.; Yu, W.; Liu, G. Flexible Janus Nanofibers: Facile Electrospinning Construction and Enhanced Luminescent-Electrical-Magnetic Trifunctionality. Chempluschem 2014, 79, 690-697.

(14) Wang, Y.; Guo, B.-H.; Wan, X.; Xu, J.; Wang, X.; Zhang, Y.-P. Janus-like Polymer Particles Prepared via Internal Phase Separation from Emulsified Polymer/oil Droplets. Polymer (Guildf). 2009, 50, 3361-3369.

(15) Walther, A.; André, X.; Drechsler, M.; Abetz, V.; Müller, A. H. E. Janus Discs. J. Am. Chem. Soc. 2007, 129, 6187-6198.

(16) Wang, H.; Zhou, H.; Yang, W.; Zhao, Y.; Fang, J.; Lin, T. Selective, Spontaneous One-Way Oil-Transport Fabrics and Their Novel Use for Gauging Liquid Surface Tension. ACS Appl. Mater. Interfaces 2015, 7, 22874-22880.

(17) Tan, T.; Tian, C.; Ren, Z.; Yang, J.; Chen, Y.; Sun, L.; Li, Z.; Wu, A.; Yin, J.; Fu, H. LSPR-Dependent SERS Performance of Silver Nanoplates with Highly Stable and Broad Tunable LSPRs Prepared through an Improved Seed-Mediated Strategy. Phys.
Chem. Chem. Phys. 2013, 15, 21034-21042. Int. Ed. 2013, 52, 341-345. Aeration. Adv. Mater. Interfaces 2016, 3, 1500774. 22762. commun. 2012, 25, 1-4. Spectroscopy. J. Phys. Chem. B 2004, 108, 3469-3473. Anal. Chim. Acta 2006, 573-574, 20-25. Chem. Soc. 2003, 125, 11502-11503. Degrad. Stab. 2010, 95, 818-825. J. Am. Chem. Soc. 2008, 130, 7812-7813. Am. Chem. Soc. 2008, 130, 11711-11718. 12814-12822.

Meilikhov, M.; Furukawa, S.; Hirai, K.; Fischer, R. A.; Kitagawa, S. Binary Janus Porous Coordination Polymer Coatings for Sensor Devices with Tunable Analyte Affinity. Angew. Chemie -

Ibañez, D.; Garoz-Ruiz, J.; Plana, D.; Heras, A.; Fermín, D. J.; Colina, A. Spectroelectrochemistry at Free-Standing Carbon Nanotubes Electrodes. Electrochim. Acta 2016, 217, 262-268.

Yang, H.-C.; Hou, J.; Wan, L.-S.; Chen, V.; Xu, Z.-K. Janus Membranes with Asymmetric Wettability for Fine Bubble

Han, D.; Xiao, P.; Gu, J.; Chen, J.; Cai, Z.; Zhang, J.; Wang, W.; Chen, T. Polymer Brush Functionalized Janus Graphene Oxide/chitosan Hybrid Membranes. RSC Adv. 2014, 4, 22759-

Garoz-Ruiz, J.; Palmero, S.; Ibañez, D.; Heras, A.; Colina, A. Press-Transfer Optically Transparent Electrodes Fabricated from Commercial Single-Walled Carbon Nanotubes. Electrochem.

Heras, A.; Colina, A.; Ibañez, D. Bipolar Spectroelectrochemistry. Anal. Chem. 2017, 89, 3879-3883.

Garoz-Ruiz, J.; Heras, A.; Palmero, S.; Colina, A. Development of a Novel Bidimensional Spectroelectrochemistry Cell Using Transfer Single-Walled Carbon Nanotubes Films as Optically Transparent Electrodes. Anal. Chem. 2015, 87, 6233-6239.

Branca, C.; Frusteri, F.; Magazù, V.; Mangione, A. Characterization of Carbon Nanotubes by TEM and Infrared

Baba, A.; Kanetsuna, Y.; Sriwichai, S.; Ohdaira, Y.; Shinbo, K. Nanostructured Carbon Nanotubes / Copper Phthalocyanine Hybrid Multilayers Prepared Using Layer-by-Layer SelfAssembly Approach. Thin Solid Films 2010, 518, 2200-2205.

Muñoz, E.; Colina, A.; Heras, A.; Ruiz, V.; Palmero, S.; LópezPalacios, J. Electropolymerization and Characterization of Polyaniline Films Using a Spectroelectrochemical Flow Cell.

Thiyagarajan, M.; Samuelson, L. A.; Kumar, J.; Cholli, A. L. Helical Conformational Specificity of Enzymatically Synthesized Water-Soluble Conducting Polyaniline Nanocomposites. J. Am.

Hintz, H.; Egelhaaf, H.; Peisert, H.; Chassé, T. Photo-Oxidation and Ozonization of Poly (3-Hexylthiophene) Thin Fi Lms as Studied by UV / VIS and Photoelectron Spectroscopy. Polym.

Zhang, Y.; Tajima, K.; Hirota, K.; Hashimoto, K. Synthesis of All-Conjugated Diblock Copolymers by Quasi-Living Polymerization and Observation of Their Microphase Separation.

Zhou, Z.; Chen, X.; Holdcroft, S. Stabilizing Bicontinuous Nanophase Segregation in $\pi \mathrm{CP}-\mathrm{C} 60$ Donor-Acceptor Blends. $J$.

Aaronson, B. D. B.; Garoz-Ruiz, J.; Byers, J. C.; Colina, A.; Unwin, P. R. Electrodeposition and Screening of Photoelectrochemical Activity in Conjugated Polymers Using Scanning Electrochemical Cell Microscopy. Langmuir 2015, 31,

Endrődi, B.; Samu, G. F.; Azam, M. A.; Janáky, C.; Visy, C. Electrochemical Synthesis and Characterization of poly(3Hexylthiophene)/single-Walled Carbon Nanotube Array Hybrid Materials. J. Solid State Electrochem. 2016, 20, 3179-3187. 


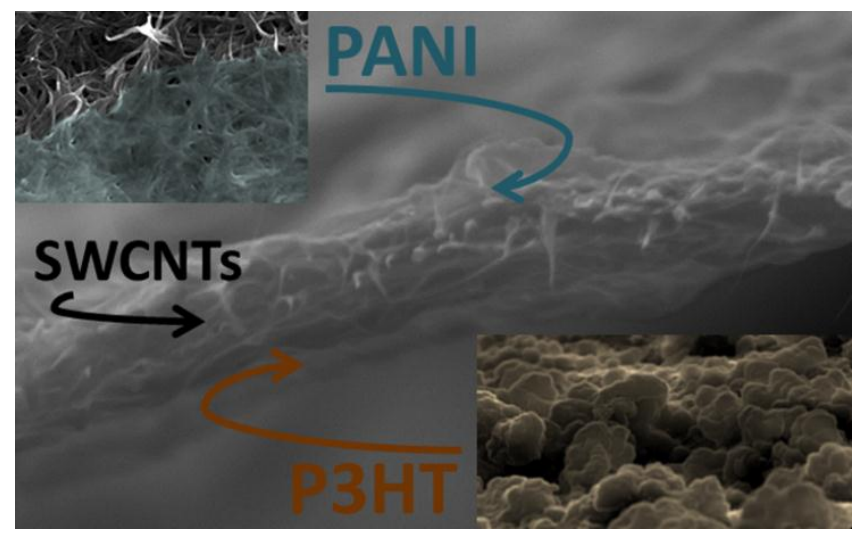

